\title{
Marked biological differences between insecticide resistant and susceptible strains of Anopheles funestus infected with the murine parasite Plasmodium berghei
}

T Mike Lo ${ }^{1,2}$ and Maureen Coetzee $e^{1,2^{*}}$

\begin{abstract}
Background: Anopheles funestus is one of the major malaria vectors in Africa but research on this species has been restricted due to the lack of viable laboratory colonies. The vectorial capacity of natural populations of An. funestus is well known but its ability to host Plasmodium in the laboratory and the development cycle of the parasite within this mosquito species was, until very recently, unknown. In this study we compared laboratory strains of An. funestus that were resistant and susceptible to pyrethroid insecticides, for their receptiveness to infection with Plasmodium berghei and compared development times with other vector species available in our laboratory.
\end{abstract}

Methods: The murine parasite $P$. berghei was used to infect two base An. funestus colonies (FANG and FUMOZ) and two selected sub-colonies with different degrees of pyrethroid resistance (FUMOZ-BS susceptible and FUMOZ-R resistant). Results were compared with the G3 strain of An. gambiae.

Results: While all colonies were able to support the parasite, the development time in An. funestus was generally longer than that recorded in the laboratory strain of An. gambiae. Infected females were able to initiate new rounds of infection when feeding on healthy mice. The pyrethroid resistant strain FUMOZ-R supported the lowest numbers of oocysts and sporozoites while the insecticide susceptible strain FUMOZ-BS produced one of the highest sporozoite indices ever documented in $P$. berghei research. The FUMOZ base colony, exhibiting partial insecticide resistance was the median in terms of infection intensity. The oocyst number in all colonies did not fully correlate with the sporozoite index, indicating possible factors influencing the sporozoites' transit from the midgut to the salivary glands.

Conclusions: The presence of both insecticide resistance and limited parasite infection phenotypes in the same individuals suggests there may be association between the two mechanisms, but further elucidation is required.

\section{Background}

Malaria remains the most serious tropical infectious disease, causing an estimated 660,000 deaths worldwide in 2010, of which 91\% occurred in Sub-Saharan Africa [1]. The disease is caused by parasites of the Plasmodium genus that are transmitted to humans by anopheline mosquitoes. Anopheles gambiae s.s., An. coluzzii, An. arabiensis and

\footnotetext{
* Correspondence: Maureen.coetzee@wits.ac.za

'Wits Research Institute for Malaria, School of Pathology, Faculty of Health Sciences, University of the Witwatersrand, Johannesburg, South Africa ${ }^{2}$ Division of the National Health Laboratory Service, Vector Control Reference Laboratory, Centre for Opportunistic, Tropical and Hospital Infections, National Institute for Communicable Diseases, Johannesburg, South Africa
}

An. funestus are the four major vectors of malaria in Africa and the surrounding islands [2,3]. Of these four species, An. funestus has been the most difficult to rear in captivity and this has presented a major obstacle to understanding the interaction of this species with the Plasmodium parasite. The only successful attempt at colonizing the species beyond the $\mathrm{F}_{10}$ generation thus far is by Hunt et al. [4]. Two colonies, one originating from Angola and another from Mozambique, were established over ten years ago. A pyrethroid (permethrin) resistant sub-colony of the Mozambican strain was subsequently selected [4].

The murine malaria infection system is a popular alternative to Plasmodium falciparum for vector-parasite 
research. It is relatively inexpensive, easy to maintain and has similarities to the infection process of human malaria parasites [5]. Of the four known murine malaria parasites, P. berghei is the most popular choice for research [5]. There are only two previous accounts of attempts to infect $A n$. funestus using $P$. berghei, one of them was unsuccessful [6]; probably because it was carried out before the impact of temperature on $P$. berghei sporogonic development was realized [7-9]. Furthermore, An. funestus is a highly anthropophilic mosquito, and its predilection for humans may have severely biased the feeding on mice. When the biology of $P$. berghei was fully understood, a number of mosquito species were found to be competent carriers for the murine parasite [10] and a very recent publication has shown that An. funestus is also a viable vector for $P$. berghei under the correct conditions [11]. This study used F-1 generation An. funestus from Mali, infected with a transgenic strain of $P$. berghe $i$ expressing green fluorescent protein and showed that $P$. berghei successfully completed its life cycle in this vector mosquito [11].

The present study aimed to determine the life cycle of $P$. berghei in laboratory strains of An. funestus that were selected for pyrethroid resistance and susceptibility.

\section{Methods}

\section{Mosquito strains}

The base An. funestus laboratory colony FUMOZ, originating from Mozambique and exhibiting low levels of pyrethroid resistance, was used to select two derivative colonies with different levels of resistance to permethrin: FUMOZ-R, resistant (selected previously) [4] and FUMOZBS, susceptible (derived in this study). FANG, a fully insecticide-susceptible strain originating from Angola [4] was used in the initial screening for infectivity rates but not for quantification of infections.

The permethrin susceptible FUMOZ-BS strain was selected as follows: 40 individual females were randomly chosen from the base FUMOZ colony and placed into separate egg-laying vials. The females were provided with a blood meal every 2-3 days until death. Egg batches were reared separately to adulthood. Half of the resulting adults from each egg batch were exposed to $4 \%$ permethrin for one hour and mortality recorded after 24 hours. Those batches giving $>85 \%$ mortality were pooled together in a cage for mating. At the 10th generation the process was repeated to ensure susceptibility was maintained. All strains were maintained at standard insectary conditions of $25 \pm 2^{\circ} \mathrm{C}, 80 \pm 10 \%$ relative humidity, with 12 hours day/night cycle including 30 minutes transition dawn/ dusk periods [4].

Seventy to ninety females were collected from each strain for each infection cohort and provided with a $10 \%$ sucrose solution for 10-14 days. To improve the rate of blood feeding, the sucrose solutions were removed 18-24 hours prior to their first infected blood meal.

The G3 strain of An. gambiae was used as a positive control for the infection process. The An. gambiae cohorts were maintained under the same conditions as An. funestus, but only 50-60 females were collected for each infection. Feeding of An. gambiae on infected mice occurred 3-4 days after eclosion as described above.

\section{Laboratory infection of mice with $P$. berghei and mosquito blood feeding}

C57/Black strain mice were injected intra-peritoneally (IP) with $0.2-0.3 \mathrm{ml}$ of $P$. berghei infected bloodstock with at least 10\% parasitaemia [5] (ANKA strain; courtesy of R. Sinden, Imperial College; also obtained from MR4 resources). The mice were then maintained under standard conditions $\left(20 \pm 1^{\circ} \mathrm{C}, 50 \%\right.$ relative humidity) and provided with food and water. The infection in the mice was monitored using Giemsa stained blood smear slides [5]. A differential analysis was performed under light microscopy at $1000 \times$ magnification. At least 10 fields of view were counted per slide to calculate the percentage of infected red blood cells. Parasitaemia levels were recorded at least every alternate day from day 3 of the infection [5]. Three to four days after initial infection, 2-4 mice were anaesthetized and the starved mosquitoes allowed to feed for up to 30 minutes. The adults that did not take a blood meal were removed from the experiment. Thereafter, the fed mosquitoes were maintained at $20 \pm 1^{\circ} \mathrm{C}, 80 \pm 10 \%$ humidity. The females were refed with an uninfected blood meal 5-7 days after the first infected blood meal to enhance the infection.

Ethical clearance for the use of mice for P. berghei infection was obtained from the animal ethics committee of the National Institute for Communicable Diseases (ethical clearance number: 110/07, 2007).

\section{Monitoring of $P$. berghei infection in mosquito specimens} Female mosquitoes were dissected to assess the presence of oocysts and sporozoites in the midgut and salivary glands, respectively [5]. Midgut dissections began 8 days post infection (pi), while salivary gland dissections began 14 days pi. For each dissection, 10-15 females per cohort were used. The dissected midguts were suspended in phosphate buffered saline (PBS) on a glass slide under a coverslip. The midguts were examined for oocysts under 100-400× magnification, and the number of oocysts present were counted and recorded. The dissected salivary glands, also suspended in PBS on a glass slide under a coverslip, were ruptured to determine the presence of sporozoites (100-400× magnification). Where possible, the sporozoites were counted to quantify the degree of infection. The remainder of the females in cohorts with confirmed infection were allowed to feed on healthy mice to determine 
Table 1 Comparison of feeding and infection rates, as well as the average oocyst and sporozoite loads of $P$. berghei in An. funestus strains and other anopheline species

\begin{tabular}{|c|c|c|c|c|c|}
\hline $\begin{array}{l}\text { Colony/species } \\
\text { (Total number of mosquitoes used) }\end{array}$ & $\begin{array}{l}\text { Feeding rate } \\
(\%)\end{array}$ & $\begin{array}{c}\text { Infection rate } \\
(\%)\end{array}$ & $\begin{array}{c}\text { Average oocyst numbers } \\
\text { (range) }\end{array}$ & $\begin{array}{c}\text { Average sporozoite numbers } \\
\text { (range) }\end{array}$ & References \\
\hline FANG $(n=567)$ & 30.3 & 4.7 & - & - & This study \\
\hline FUMOZ $(n=1968)$ & 52.1 & 20.5 & 24 & 1573 & This study \\
\hline FUMOZ-R $(n=2139)$ & 46.7 & 6 & 6 & 1586 & This study \\
\hline FUMOZ-BS $(n=2023)$ & 50.5 & 15.5 & 53 & 7249 & This study \\
\hline An. dureni & - & 15.2 & $60-80$ & 7400 & Yoeli 1965 [10] \\
\hline An. quadrimaculatus & - & 42.5 & $100-500$ & $100-300$ & Yoeli et al. 1965 [12] \\
\hline An. stephensi & - & 50.5 & $60-80$ & $6840-8200$ & Yoeli et al. 1965 [12] \\
\hline An. funestus trial 1 & - & 62.5 & $47(1-141)$ & - & Xu et al. 2013 [11] \\
\hline trial 2 & - & 95.0 & $120(1-347)$ & - & \\
\hline
\end{tabular}

the infectivity of the sporozoites, and to complete the infection cycle [5]. The mice infected via the mosquitoes were maintained and monitored in the same manner as the IP-infected mice. Two-sample T-tests and one-way ANOVA were used to compare the feeding and infection rates between the strains, as well as to compare the oocyst and sporozoite numbers.

\section{Results}

Both $A n$. funestus base colonies were susceptible to $P$. berghei infection, but at different rates (Table 1, Figure 1). FUMOZ mosquitoes exhibited a higher feeding success rate, with over $50 \%$ of the females $(n=1968)$ from 25 cohorts taking a blood meal, similar to the feeding rate seen in An. gambiae G3 strain where an average of $51.9 \%$ of the females took a blood meal. The insecticide susceptible FANG cohorts exhibited lower feeding success rate, with only $28-36 \%$ of the 567 females from 10 cohorts taking a blood meal. The FUMOZ cohorts also had higher infection rates. On average, $20 \%$ of fed females became infective, but some cohorts produced over $30 \%$ infection rates.
Infection in FANG females was low and never exceeded $8 \%$ in any of the 10 cohorts. The An. gambiae G3 infection rates averaged $35.8 \%$, ranging from $10.7-48.2 \%$. The feeding and infection rates between FANG and FUMOZ were significantly different $(\mathrm{p}<0.01)$. The feeding rates between FUMOZ and its derivative strains were not significantly different $(p>0.01)$ but the infection rates were $(\mathrm{p}<0.01)$ (Figure 1).

Midgut dissections revealed oocysts 10 days pi at the earliest, with mature oocysts observed as late as 20 days pi (Figure 2). The average time for oocysts to appear was 14 days. Oocysts were not restricted to any specific region of the midgut, although they were more often located on the posterior half. Mature oocysts were $40 \pm 4 \mu \mathrm{m}$ in diameter with sporozoites easily observed (Figures 2 and 3).

Sporozoites were detected in the salivary glands as early as day 16 after the initial infected blood meal but this occurred in less than $1 \%$ of the infected females. The sporozoites were more consistently detected after 18-21 days of development in all colonies, and sometimes only appeared as late as day 24. The mature, infective

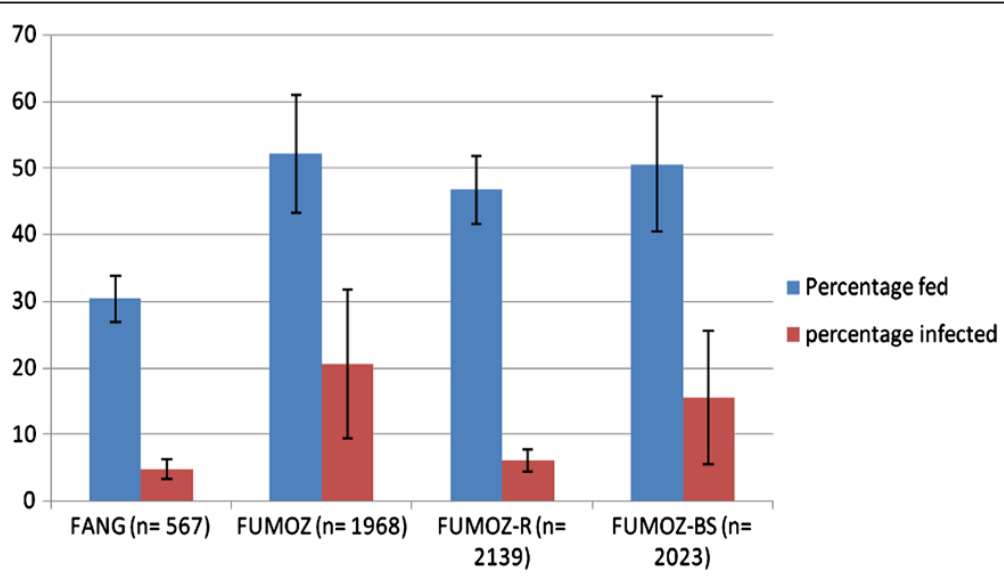

Figure 1 Feeding and infection rates of Anopheles funestus colonies. 


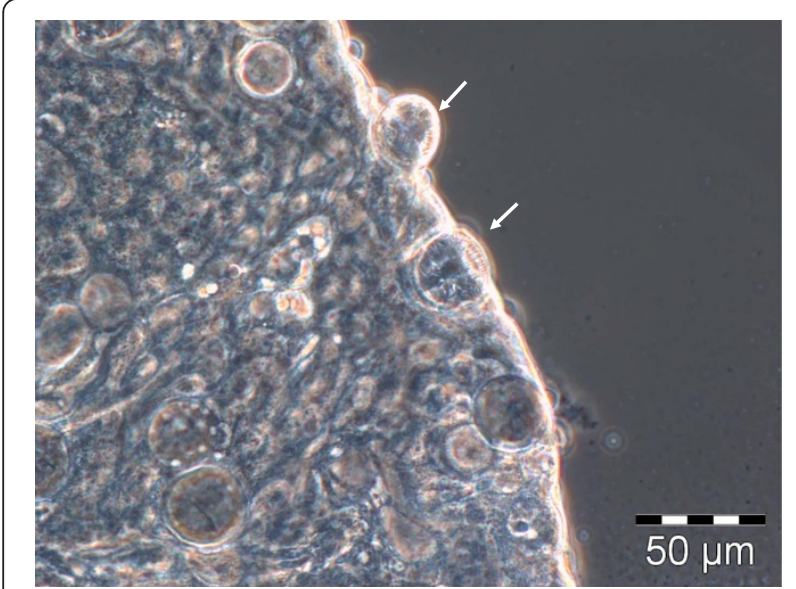

Figure $\mathbf{2}$ Oocysts on An. funestus midgut at $\mathbf{2 0}$ days post infected blood meal. The oocysts are at different stages of maturation, some containing immature sporozoites (white arrows) (phase contrast, $400 \times$ magnification).

sporozoites found in the salivary glands were $13 \pm 2 \mu \mathrm{m}$ in length, and had the classical crescent shape associated with malaria sporozoites (Figure 4).

By comparison, the development of $P$. berghei in An. gambiae was faster under the same conditions, typically by $2-3$ days. Oocysts were observed as early as 8 days, but on average appeared 11-12 days after infection. The earliest point when sporozoites were observed was on day 14, but was more consistently detected from day 16-20 (Figure 5).

Quantitative comparison of $P$. berghei developmental stages in the three FUMOZ strains (Tables 2 and 3 ) showed that FUMOZ-BS was most susceptible to P. berghei, being able to host at least 30 oocysts and carry on average over 7,000 sporozoites in its salivary glands. Oocyst and

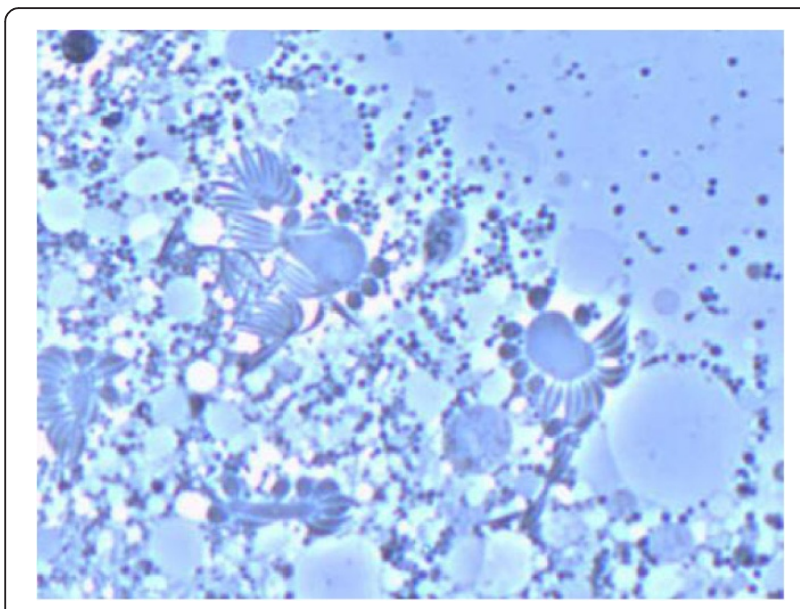

Figure 3 Sporozoite assembly around blastophores, from ruptured oocysts in the midgut. The sporozoites are all immature and at different stages of development (phase contrast, $400 \times$ magnification).

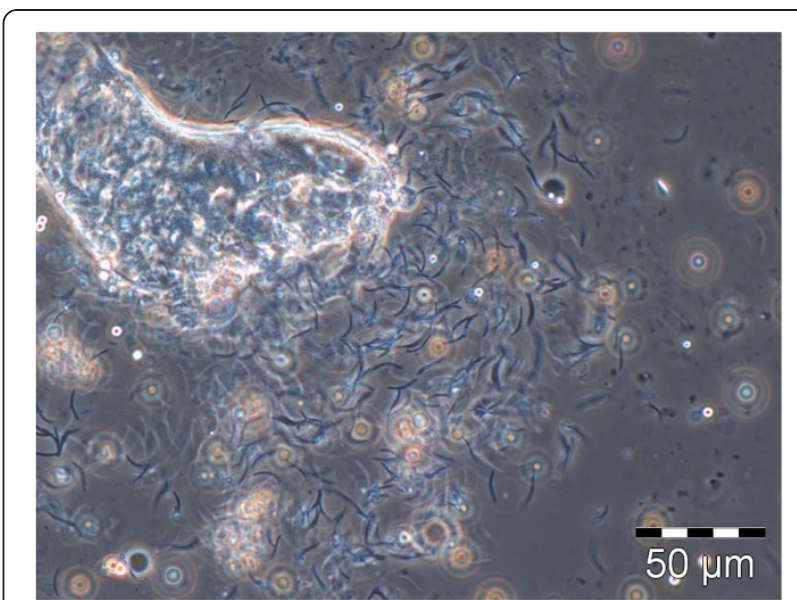

Figure 4 Ruptured An. funestus salivary gland showing mature crescent sporozoites of $P$. berghei (phase contrast, $400 \times$ magnification).

sporozoite numbers in FUMOZ-R were both much lower compared with the other two strains (Tables 2 and 3). A more detailed breakdown of the oocyst distribution showed that infected FUMOZ females carried a highly variable number of oocysts (ranging from 1 to 100), FUMOZ-R never supported more than 10 oocysts per female and over $60 \%$ FUMOZ-BS females contained more than 50 oocysts (Table 2). A similar breakdown of the sporozoite distribution showed an evenly distributed range of sporozoite numbers up to 10,000 in FUMOZ. In FUMOZ-R the number never exceeded 5,000 while FUMOZ-BS had $46.1 \%$ of the females with more than 10,000 sporozoites (Table 3).

Infection of healthy mice using infected An. funestus was carried out 21 days or later after an infected blood meal. Transmission of the parasite by An. funestus led to $84.2 \%$ of the mice becoming infected $(\mathrm{n}=19)$, and all infected mice died within 9 days following cyclical transmission.

\section{Discussion}

Plasmodium berghei is a popular alternative model organism in malaria research, but for over a decade after its discovery [13] there were only two isolated successes of infection in the laboratory [7]. Anopheles funestus was classified as a non-vector for the parasite during this time [6]. When lower temperatures were shown to be essential for $P$. berghei sporogonic development and invasion of the salivary glands [8-10], the vectorial capacity of An. funestus was not reassessed until very recently [11]. The lack of a laboratory colony and the highly anthropophilic nature of this species were possible factors contributing to its non-vector classification through the decades. However, the recent work carried out in Mali [11], and the current successful infection of An. funestus under 


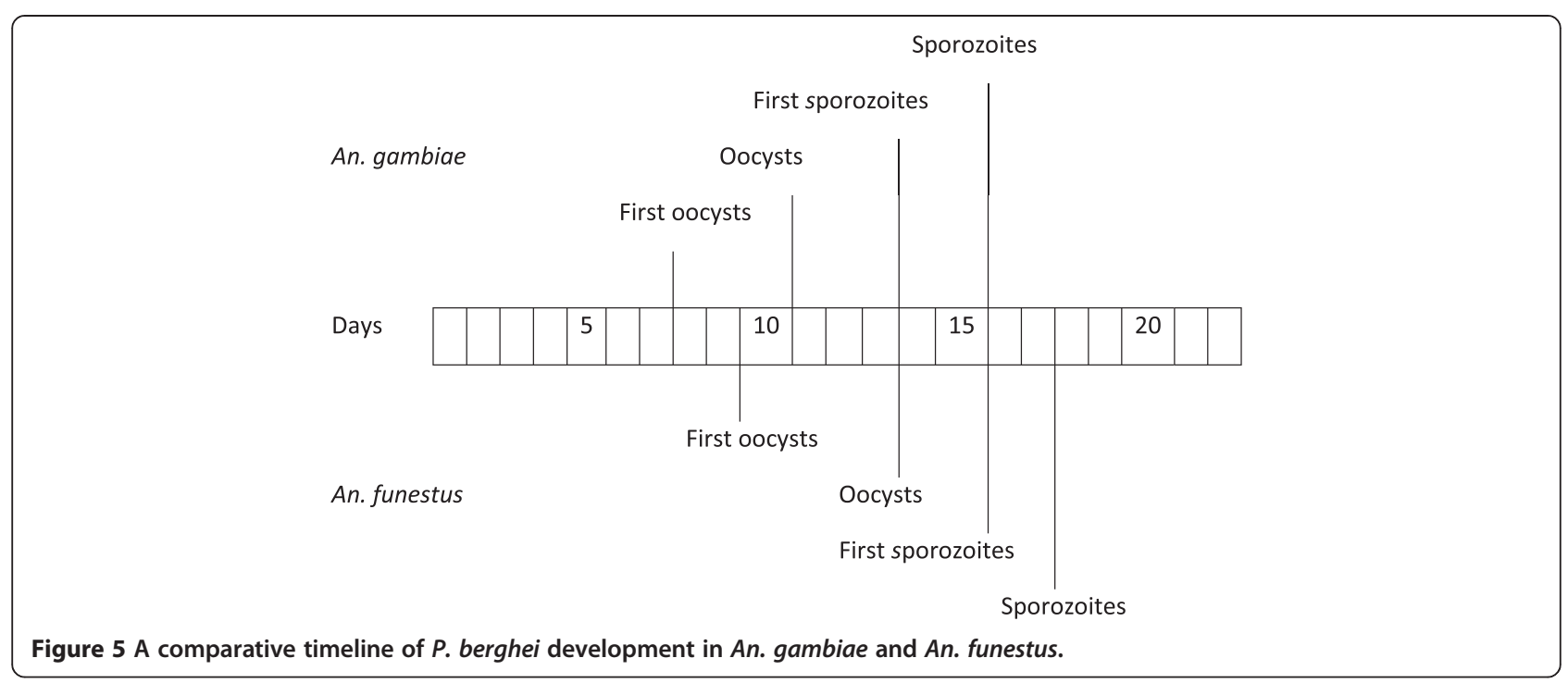

the correct conditions indicate that this species is in fact a viable vector for $P$. berghei.

In the present study, the parasite displayed significantly different developmental times in An. funestus compared with other known $P$. berghei experimental vectors. The FUMOZ An. funestus females would only feed on mice with consistency 10-14 days after eclosion, significantly later than other anophelines (e.g. An. quadrimaculatus can be used for infection experiments 2-3 days after eclosion [7]) and twice the time recorded for the An. funestus from Mali [11]. The development time for the parasite was also delayed compared with other mosquitoes. In its natural vector, $A n$. dureni, and the Asian malaria vector $A n$. stephensi, P. berghei requires 10-11 days to complete sporogonic development [10,12], while An. quadrimaculatus is somewhat slower, requiring 1314 days [14]. It is possible to observe sporozoites in An. stephensi salivary glands as early as nine days postinfection and on day ten for An. quadrimaculatus [8,9]. In $A n$. funestus, the earliest salivary gland invasions were observed at 16 days post-infection, but sporozoites were more consistently seen in the salivary glands 1821 days after infection. This is consistent with results for An. funestus from Mali [11].

Once the sporozoites had successfully invaded the salivary glands, the female An. funestus carried sporozoites in the salivary glands for the remainder of her life.
Successful infection of healthy mice was achieved using infected An. funestus females 60 days after eclosion.

Both unselected An. funestus colonies, FANG and FUMOZ, were susceptible to $P$. berghei infection. However, FANG had both a low feeding and infection rate, making it less useful for experimental purposes. FUMOZ was thus the strain of choice for establishing the infection system. In an effort to resolve whether the partial insecticide resistance phenotype of FUMOZ had an impact on its vectorial capacity, two sub-colonies with varying permethrin resistance were also tested for infectivity. All three colonies had similar feeding rates but the infection rate was highly variable. FUMOZ-R, exhibiting high levels of permethrin resistance [4], was the most resistant to parasite infection, with low oocyst and sporozoite counts. Although the oocyst load in FUMOZ-R was amongst the lowest detected in experimental vectors $[7,10,12]$, considerable numbers of sporozoites were still able to migrate to the salivary glands, thus maintaining its viability for parasite infection. FUMOZ-BS, selected for the pyrethroid susceptible phenotype, was highly susceptible to $P$. berghei infection, typically carrying a modest oocyst load. Although the oocyst load in FUMOZ-BS is not high compared with the frequently used experimental vector An. stephensi, the sporozoite index in the An. funestus strain is amongst the highest recorded using the murine parasite $[10,15]$. The FUMOZ-BS sporozoite load in this

Table 2 Comparison of oocyst load in 13-15 day post-infected females from the FUMOZ sub-strains

\begin{tabular}{ccccc}
\hline Colony & Average oocyst numbers & \multicolumn{3}{c}{ Oocyst numbers } \\
\cline { 3 - 5 } (number of infected mosquitoes dissected) & $25(1-60)$ & $\mathbf{1 - 1 0}$ & $\mathbf{1 1 - 3 0}$ & $\mathbf{3 1 - 5 0}$ \\
\hline FUMOZ $(n=59)$ & $8(1-15)$ & $58 \%$ & $10 \%$ & 0 \\
FUMOZ-R $(n=64)$ & $55(27-83)$ & 0 & $18 \%$ & 0 \\
FUMOZ-BS $(n=65)$ & & 0 & $32 \%$ \\
\hline
\end{tabular}


Table 3 Comparison of sporozoite load in 18-21 day post-infected females from the FUMOZ sub-strains

\begin{tabular}{|c|c|c|c|c|c|c|}
\hline \multirow{2}{*}{$\begin{array}{c}\text { Colony } \\
\text { (number of infected mosquitoes dissected) }\end{array}$} & \multirow{2}{*}{$\begin{array}{l}\text { Average sporozoite } \\
\text { numbers (range) }\end{array}$} & \multicolumn{5}{|c|}{ Sporozoite numbers } \\
\hline & & $1-1000$ & $1001-2000$ & $2001-5000$ & $5001-10000$ & $10000-15000$ \\
\hline FUMOZ $(n=59)$ & $2248(1075-3421)$ & $20 \%$ & $30 \%$ & $30 \%$ & $20 \%$ & 0 \\
\hline FUMOZ-R $(n=64)$ & $1586(542-2630)$ & $27 \%$ & $53 \%$ & $25 \%$ & 0 & 0 \\
\hline FUMOZ-BS $(n=65)$ & 7648 (2662-12634) & $7.7 \%$ & $15.4 \%$ & $7.7 \%$ & $23.1 \%$ & $46.1 \%$ \\
\hline
\end{tabular}

study surpassed that observed in $P$. berghei's natural vector, An. dureni [10]. The unselected FUMOZ base strain was the median, with fairly light oocyst growth and highly variable sporozoite numbers.

Although oocyst load in An. funestus usually corresponded with sporozoite index, it was not an absolute correlation and some discrepancies were observed where low oocyst numbers $(<10)$ led to heavy sporozoite infections $(>2,000)$ as in the case of FUMOZ-R. Conversely, the sporozoite numbers observed in FUMOZ were sometimes lower than expected given that $32 \%$ of the females had $>50$ oocysts present. It is unclear what factors affect the transit of the sporozoites between the midgut and salivary glands and this will require further investigation.

The presence of resistance to insecticide and low parasite infectivity in the FUMOZ-R (and conversely, susceptibility to both factors in FUMOZ-BS) suggests that there may be some association between these two phenotypes. There are currently no known genes or pathways that act in both insecticide resistance and parasite infection in anophelines. The most comparable model is Culex quinquefasciatus and the lymphatic filariasis parasite, Wuchereria bancrofti. Insecticide resistant $C x$. quinquefasciatus exhibiting increased esterase activity, affects the development of $W$. bancrofti larvae possibly by arresting the parasites in the gut cells [16]. The correlation was easier to quantify in $C x$. quinquefasciatus as the esterase amplicon duplication is the dominant resistant mechanism, found in over $80 \%$ of insecticide resistant $C x$. quinquefasciatus [17]. The phenomenon may be more difficult to correlate between the two phenotypes in anophelines, since malaria vectors employ a number of insecticide resistance mechanisms [18-22] that are involved in different manners depending on the type of insecticide the mosquitoes are exposed to. However, the pyrethroid resistance in southern African An. funestus has been well-studied showing that mono-oxygenase detoxifying enzymes in the CYP6 class are mainly responsible [23-27]. It is possible that these enzymes are preventing $P$. berghei parasite development in An. funestus similar to the esterase enzymes in $C x$. quinquefasciatus inhibiting $W$. bancrofti development.

\section{Conclusions}

The results indicate that metabolic insecticide resistance plays a role in determining the infectivity of An. funestus to $P$. berghei parasites with resistant strains being less condusive to parasite development than susceptible strains. Further investigations are needed to determine whether infectivity of the vectors is influenced by their insecticide resistance profile in natural populations with respect to Plasmodium falciparum as this may have implications for future vector control interventions.

\section{Competing interests}

The authors declare that they have no competing interests.

\section{Authors' contributions}

TML carried out the experiments and drafted the manuscript. MC conceived the project and assisted with the drafting of the manuscript. Both authors read and approved the final version of the manuscript.

\section{Acknowledgements}

We thank P. Hawkins, I. Linnekrugel and T. Marumo for assistance with the animals used in the study. Z. Zulu, Z. Mnisi, B. Spillings and O. Wood are thanked for their assistance with mosquito colony maintenance. Prof R. Sinden and Dr A. Blagborough from Imperial College are thanked for their advice in setting up the infections in mice. Partial funding was provided through the South African Malaria Initiative of the Department of Science and Technology. MC is funded by the South African DST/NRF Research Chair Initiative.

Received: 16 May 2013 Accepted: 11 June 2013

Published: 19 June 2013

\section{References}

1. WHO: World malaria report 2012. Geneva, Switzerland; 2012. http://www. who.int/malaria/publications/world_malaria_report_2012/en/index.html.

2. Gillies MT, De Meillon B: The Anophelinae of Africa south of the Sahara. Publ Sth Afr Inst Med Res 1968, 54:1-343.

3. Coetzee M, Hunt RH, Wilkerson R, Della Torre A, Coulibaly MB, Besansky NJ: Anopheles coluzzii and Anopheles amharicus, new members of the Anopheles gambiae complex. Zootaxa 2013, 3619:246-274.

4. Hunt RH, Brooke BD, Pillay C, Koekemoer LL, Coetzee M: Laboratory selection for and characteristics of pyrethroid resistance in the malaria vector Anopheles funestus. Med Vet Entomol 2005, 19:271-275.

5. Sinden RE, Butcher GA, Beetsma AL: Maintenance of the Plasmodium berghei life cycle. In Methods in Molecular Medicine. 72nd edition. Edited by Doolan DL. USA: Humana Press; 2002:25-40.

6. Van den Berghe L: The history of the discovery of Plasmodium berghei. Indian J Malariol 1954, 8:241-243.

7. Yoeli M, Most H: The biology of a newly isolated strain of Plasmodium berghei in a rodent host and in experimental mosquito vectors. Trans Roy Soc Trop Med Hyg 1960, 54:549-555.

8. Vanderberg JP, Yoeli M: Some physiological and metabolic problems related to maintenance of the Plasmodium berghei cycle in Anopheles quadrimaculatus. Ann Soc Belges Med Trop 1965, 45:419-426.

9. Vanderberg JP, Yoeli M: Effects of temperature on sporogonic development of Plasmodium berghei. J Parasitol 1966, 52:559-566.

10. Yoeli M: Studies on Plasmodium berghei in nature and under experimental conditions. Trans Roy Soc Trop Med Hyg 1965, 59:255-276.

11. Xu J, Hillyer JF, Coulibaly B, Sacko M, Dao A, Niare O, Riehle MM, Traore SF, Vernick KD: Wild Anopheles funestus mosquito genotypes are permissive for infection with the rodent malaria parasite Plasmodium berghei. PLoS One 2013, 8(4):e61181. 
12. Yoeli M, Vanderberg JP, Nawrot R, Most H: Studies on sporozoite-induced infections of rodent malaria II. Anopheles stephensi as an experimental vector of Plasmodium berghei. Am J Trop Med Hyg 1965, 14:927-930.

13. Vincke $\mathrm{H}$, Lips M: Un nouveau plasmodium d'un rongeus sauvage du Congo, Plasmodium berghei n. sp. Ann Soc Belge Méd Trop 1948, 28:97-104.

14. Yoeli M, Most H, Bone G: Plasmodium berghei: cyclical transmission by experimentally infected Anopheles quadrimaculatus. Science 1964, 144(3626):1580-1581.

15. Sinden RE, Dawes EJ, Alavi Y, Waldock J, Finney O, Mendoza J, Butcher GA, Andrews L, Hill AV, Gilbert SC, Basáñez M: Progression of Plasmodium berghei through Anopheles stephensi is density-dependent. PLoS Pathogen 2007, 3(12):e195.

16. McCarroll L, Paton MG, Karunaratne SH, Jayasuryia HT, Kalpage KS, Hemingway J: Insecticides and mosquito-borne disease. Nature 2000, 407:961-962.

17. Hemingway J, Karunaratne SH: Mosquito carboxylesterases: a review of the molecular biology and biochemistry of a major insecticide resistance mechanism. Med Vet Entomol 1998, 12:1-12.

18. Nikou D, Ranson H, Hemingway J: An adult-specific CYP6 P450 gene is over expressed in a pyrethroid-resistant strain of the malaria vector, Anopheles gambiae. Gene 2003, 318:91-102.

19. Ranson H, Jensen B, Vulule JM, Wang X, Hemingway J, Collins FH: Identification of a point mutation in the voltage-gated sodium channel gene of Kenyan Anopheles gambiae associated with resistance to DDT and pyrethroids. Insect Mol Biol 2000, 9:491-497.

20. Ranson H, Rossiter L, Ortelli F, Jensen B, Wang X, Roth CW, Collins FH, Hemingway J: Identification of a novel class of insect glutathione S-transferases involved in resistance to DDT in the malaria vector Anopheles gambiae. Biochem J 2001, 359:295-304.

21. Ranson H, Paton MG, Jensen B, McCarroll L, Hemingway J, Collins FH: Genetic mapping of genes conferring permethrin resistance in the malaria vector Anopheles gambiae. Insect Mol Biol 2004, 13:379-386.

22. Weill M, Lutfalla G, Mogensen K, Chandre F, Berthomieu A, Berticat C, Pasteur N, Philips A, Fort P, Raymond M: Comparative genomic: insecticide resistance in mosquito vectors. Nature 2003, 423:136-137.

23. Amenya DA, Koekemoer LL, Vaughan A, Morgan JC, Brooke BD, Hunt RH, Ranson H, Hemingway J, Coetzee M: Isolation and sequence analysis of P450 genes from a pyrethroid resistant colony of the major malaria vector Anopheles funestus. DNA Sea 2005, 16:437-445.

24. Amenya DA, Naguran R, Lo TC, Ranson H, Spillings BL, Wood OR, Brooke BD, Coetzee M, Koekemoer LL: Over expression of a cytochrome P450 (CYP6P9) in a major African malaria vector, Anopheles funestus, resistant to pyrethroids. Insect Mol Biol 2008, 2008(17):19-25.

25. Brooke BD, Kloke G, Hunt RH, Koekemoer LL, Temu EA, Taylor ME, Small G, Hemingway J, Coetzee M: Bioassay and biochemical analyses of insecticide resistance in southern African Anopheles funestus (Diptera: Culicidae). Bull Entomol Res 2001, 91:265-272.

26. Christian R, Strode C, Ranson H, Coetzer N, Coetzee M, Koekemoer LL: Microarray analysis of a pyrethroid resistant African malaria vector, Anopheles funestus, from southern Africa. Pestic Biochem Physiol 2011, 99:140-147.

27. Wondji CS, Irving H, Morgan J, Lobo NF, Collins FH, Hunt RH, Coetzee M, Hemingway J, Ranson H: Two duplicated P450 genes are associated with pyrethroid resistance in Anopheles funestus, a major malaria vector. Genome Res 2009, 19:452-459.

doi:10.1186/1756-3305-6-184

Cite this article as: Lo and Coetzee: Marked biological differences between insecticide resistant and susceptible strains of Anopheles funestus infected with the murine parasite Plasmodium berghei. Parasites \& Vectors 2013 6:184

\section{Submit your next manuscript to BioMed Central and take full advantage of:}

- Convenient online submission

- Thorough peer review

- No space constraints or color figure charges

- Immediate publication on acceptance

- Inclusion in PubMed, CAS, Scopus and Google Scholar

- Research which is freely available for redistribution

Submit your manuscript at www.biomedcentral.com/submit
Ciomed Central 